\title{
Sum Rate Optimization of IRS-Aided Uplink Muliantenna NOMA with Practical Reflection
}

This paper was downloaded from TechRxiv (https://www.techrxiv.org).

LICENSE

CC BY-NC-SA 4.0

SUBMISSION DATE / POSTED DATE

27-01-2022 / 01-02-2022

\section{CITATION}

Choi, Jihyun; Cantos, Luiggi; Choi, Jinho; Kim, Yun Hee (2022): Sum Rate Optimization of IRS-Aided Uplink Muliantenna NOMA with Practical Reflection. TechRxiv. Preprint.

https://doi.org/10.36227/techrxiv.19076315.v1

$\mathrm{DOI}$

10.36227/techrxiv.19076315.v1 


\title{
Sum Rate Optimization of IRS-Aided Uplink Muliantenna NOMA with Practical Reflection
}

\author{
Jihyun Choi, Luiggi Cantos, Jinho Choi, and Yun Hee Kim
}

\begin{abstract}
In this letter, we consider intelligent reflecting surface (IRS) aided nonorthogonal multiple access (NOMA) for the uplink employing multiple receive antennas in order to achieve high spectral efficiency and massive connectivity. In particular, the phase shifts of the IRS are optimized under a generalized reflection model to maximize the sum rate. For the unit modulus reflection, a determinant-maximization problem is formulated and solved through extended semidefinite relaxation (max-det). For the practical reflection, we apply the limited memory BroydenFletcher-Goldfarb-Shanno (L-BFGS) algorithm by deriving the gradient of the complicated objective function. Based on the results, we observe that the max-det solution provides a nearoptimal performance but at high complexity for a large number $N$ of IRS elements, while the L-BFGS relieves the complexity issue for a large $N$ and provides a performance comparable to or better than a conventional sequential optimization at a reduced computational time.
\end{abstract}

Index Terms-Intelligent reflecting surface, nonorthogonal multiple access, practical reflection, multiple receive antennas

\section{INTRODUCTION}

Intelligent reflecting surfaces (IRSs) have drawn enormous attention from academy and industry as a cost-effective building block for 6th generation $(6 \mathrm{G})$ networks demanding high spectral and energy efficiency [1]. An IRS composed of large passive elements can be deployed on a surrounding object to improve propagation channels by controlling the reflecting elements. Due to the advantages of low-cost and power-efficient implementation, IRS-assisted wireless communications have been explored with various communication techniques for various purposes [2], which include attractive nonorthogonal multiple access (NOMA) for higher spectral efficiency and more massive connectivity [3], [4].

One of major concerns in realizing an IRS-aided NOMA network is how to reflect an incident superimposed NOMA signal properly to meet the design goals of a given network. In the downlink, IRS reflection optimization was studied not only for a single-antenna base station (BS) [5], [6] but also for a

Jihyun Choi and Yun Hee Kim are with the Department of Electronic Engineering, Kyung Hee University, Yongin 17104, Korea (e-mail: solbangwool_@khu.ac.kr, yheekim@khu.ac.kr).

Luiggi Cantos is with the Department of Electronics and Information Convergence Engineering, Kyung Hee University, Yongin 17104, Korea (email: 1rcantos@khu.ac.kr).

Jinho Choi is with School of Information Technology, Deakin University, Burwood, Australia (e-mail:jinho.choi@ deakin.edu.au).

This research was supported by the National Research Foundation of Korea (NRF) under Grant NRF-2021R1A2C1005869 and by the Institute of Information \& Communications Technology Planning \& Evaluation (IITP) under the Information Technology Research Center (ITRC) support program IITP-2021-0-02046, with funding from the Ministry of Science and ICT (MSIT), Korea (Corresponding author: Yun Hee Kim). multiantenna BS performing active beamforming [7]-[11]. For the single-antenna BS, joint optimization of the IRS reflection and power allocation was studied to minimize the transmit power [5] and to optimize the sum rate [6]. For the multiantenna BS, alternating optimization (AO) algorithms of active beamforming and IRS reflection were designed to maximize the sum rate [7], to minimize the transmit power [8], [9], [11], and to maximize the minimum rate [10]. For the IRS-aided uplink NOMA, IRS reflection was optimized to maximize the sum rate with a single-antenna BS mostly without [12] and with wireless powered communication (WPC) [13], [14]. Recently, the IRS-aided uplink NOMA with a multiantenna receiver was incorporated in WPC, where two NOMA users were assisted by a different IRS with ideal reflection of both amplitude and phase controls [15].

The IRS with the unit modulus reflection has been optimized through semidefinite relaxation (SDR) dealing with the signal and interference power in a trace form [7], [10]-[14]. However, the complexity of SDR-based algorithms becomes unacceptably high for a large number $N$ of IRS elements. In the uplink NOMA with a multi-antenna receiver [15], where the sum rate takes a similar form with the multiple-input multipleoutput (MIMO) capacity, IRS optimization was performed by sequentially optimizing one element while fixing the other elements as in the IRS-aided MIMO system [16] since SDR in a form of trace maximization is no longer applicable. However, the sequential optimization incurs a long computational delay making parallel processing inapplicable. In addition, practical IRS circuits showing phase-dependent amplitudes [17] have not been considered for IRS-aided NOMA.

In this context, we consider the IRS-aided uplink NOMA supported by a multiantenna BS and a practical IRS with a generalized reflection model. The main contributions of this paper are summarized as follows:

- We formulate the sum rate maximization problem for the multi-antenna BS with successive interference cancellation (SIC) under the practical IRS reflection [17].

- We tackle the problem under the unit modulus reflection with an extended SDR approach of determinant maximization to obtain an upper bound on the optimal sum rate and to provide a feasible solution outperforming that of the conventional method [16].

- For a large $N$ and also for the practical IRS reflection, we employ the limited memory Broyden-Fletcher-GoldfarbShanno (L-BFGS) algorithm [18], [19] known to be efficient for a large-scale problem. To implement the LBFGS algorithm, we provide the gradient of the objective function incorporating the generalized reflection model. 


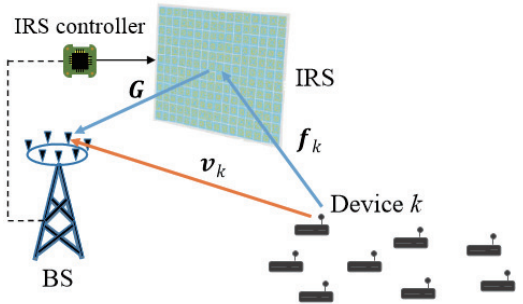

Fig. 1. IRS-aided uplink consisting of a multiantenna BS, $K$ single-antenna devices, and an IRS with $N$ elements.

Notation: We denote by $\mathbb{C}^{n \times m}$ and $\mathbb{R}^{n \times m}$ the sets of $n \times m$ complex-valued and real-valued matrices, respectively, with $\mathbb{C}^{n}=\mathbb{C}^{n \times 1}$ and $\mathbb{R}^{n}=\mathbb{R}^{n \times 1}$. The set of $n \times n$ positive semidefinite Hermitian matrices is denoted by $\mathbb{S}_{+}^{n}$. The transpose, Hermitian, and trace are denoted by $(\cdot)^{\mathrm{T}},(\cdot)^{\mathrm{H}}$, and $\operatorname{tr}(\cdot)$, respectively. We use $\operatorname{diag}(\boldsymbol{a})$ for the diagonal matrix with a diagonal vector $\boldsymbol{a}$ and $\mathcal{C N}(\boldsymbol{\mu}, \boldsymbol{\Sigma})$ for complex Gaussian distribution with mean vector $\boldsymbol{\mu}$ and covariance matrix $\boldsymbol{\Sigma}$.

\section{System Model AND PROBlem Formulation}

\section{A. System Model}

Consider the uplink of a single-cell network described in Fig. 1 that consists of a BS with $M$ antennas, $K$ single-antenna devices, and a single IRS with $N$ reflection elements. The channels from device $k$ to the BS and from device $k$ to the IRS is denoted by $\boldsymbol{v}_{k} \in \mathbb{C}^{M}$ and $\boldsymbol{f}_{k} \in \mathbb{C}^{N}$, respectively, for $k \in \mathbb{K} \triangleq\{1,2, \cdots, K\}$. The channel from the IRS to the BS is denoted by $G \in \mathbb{C}^{M \times N}$. To capture the amplitude distortion of practical IRS control circuits, the IRS reflection vector, $\boldsymbol{\theta}=\left[\theta_{1}, \theta_{2}, \cdots, \theta_{N}\right]^{\top} \in \mathbb{C}^{N}$, is modeled as [17]

$$
\theta_{n}=\beta\left(\phi_{n}\right) e^{j \phi_{n}}
$$

where

$$
\beta_{n}\left(\phi_{n}\right)=\left(1-\beta_{\min }\right)\left(\frac{\sin \left(\phi_{n}-\phi_{0}\right)+1}{2}\right)^{\alpha}+\beta_{\min }
$$

with $\alpha \geq 0, \beta_{\min } \geq 0$, and $\phi_{0} \geq 0$. These parameters are related to the specific circuit implementation, and also represent the unit modulus reflection with $\alpha=0$ and $\beta_{\min }=0$.

Since the devices transmit their symbols simultaneously for the uplink NOMA, the signal received at the BS is written as

$$
\boldsymbol{y}=\sum_{k=1}^{K} \sqrt{p_{k}}\left(\boldsymbol{G} \operatorname{diag}(\boldsymbol{\theta}) \boldsymbol{f}_{k}+\boldsymbol{v}_{k}\right) s_{k}+\boldsymbol{z},
$$

where $s_{k}$ and $p_{k}$ are the symbol and transmit power of device $k \in \mathbb{K}$, respectively, and $\boldsymbol{z} \sim \mathcal{C N}\left(\mathbf{0}_{M}, \sigma^{2} \boldsymbol{I}_{M}\right)$ is the noise vector at the BS. We can rewrite (3) as

$$
\boldsymbol{y}=\sum_{k=1}^{K} \sqrt{p_{k}} \tilde{\boldsymbol{H}}_{k} \tilde{\boldsymbol{\theta}} s_{k}+\boldsymbol{z}
$$

where $\tilde{\boldsymbol{H}}_{k}=\left[\begin{array}{ll}\boldsymbol{H}_{k} & \boldsymbol{v}_{k}\end{array}\right]$ with $\boldsymbol{H}_{k}=\boldsymbol{G} \operatorname{diag}\left(\boldsymbol{f}_{k}\right) \in \mathbb{C}^{M \times N}$ and $\tilde{\boldsymbol{\theta}}=\left[\begin{array}{ll}\boldsymbol{\theta}^{\top} & 1\end{array}\right]^{\top} \in \mathbb{C}^{N+1}$, resulting in the equivalent channel $\tilde{\boldsymbol{H}}_{k} \tilde{\boldsymbol{\theta}}$ of device $k$.
From [20], by applying the minimum mean square error (MMSE) detection with SIC, the sum rate for a given channel $\tilde{\boldsymbol{H}}_{k} \tilde{\boldsymbol{\theta}}$ is obtained irrespective of SIC ordering as

$R_{\text {sum }}(\boldsymbol{\phi}, \boldsymbol{p})=\log _{2} \operatorname{det}\left(\boldsymbol{I}_{M}+\sum_{k=1}^{K} \frac{p_{k}}{\sigma^{2}} \tilde{\boldsymbol{H}}_{k} \tilde{\boldsymbol{\theta}} \tilde{\boldsymbol{\theta}}^{\mathrm{H}} \tilde{\boldsymbol{H}}_{k}^{\mathrm{H}}\right)$,

where $\boldsymbol{\phi}=\left[\phi_{1}, \phi_{2}, \cdots, \phi_{N}\right]^{T}, \boldsymbol{p}=\left[p_{1}, p_{2}, \cdots, p_{K}\right]^{T}$, and $\tilde{\boldsymbol{\theta}}$ depends on $\phi$.

\section{B. Problem Formulation}

This paper aims at maximizing the sum rate by optimizing the transmit power and IRS phase shifts as

$$
\begin{array}{r}
\max _{\boldsymbol{\phi} \in \mathbb{R}^{N}, \boldsymbol{p} \in \mathbb{R}^{K}} R_{\text {sum }}(\boldsymbol{\phi}, \boldsymbol{p}) \\
\text { s.t. } 0 \leq \phi_{n}<2 \pi, \forall n, \\
0 \leq p_{k} \leq P_{k}, \forall k .
\end{array}
$$

In fact, the optimal power of (6) is given by $\boldsymbol{p}=\boldsymbol{P} \triangleq$ $\left[P_{1}, P_{2}, \cdots, P_{K}\right]^{\top}$ since the sum rate (5) is a non-decreasing function of $p_{k}$ irrespective of $\phi$. Hence, problem (6) is reduced to the following phase optimization problem with the objective function $\mathcal{R}(\phi) \triangleq R_{\text {sum }}(\boldsymbol{\phi}, \boldsymbol{P})$ :

$$
\begin{gathered}
\max _{\phi \in \mathbb{R}^{N}} \mathcal{R}(\boldsymbol{\phi})=\log _{2} \operatorname{det}\left(\boldsymbol{I}_{M}+\boldsymbol{S}(\boldsymbol{\phi})\right), \\
\text { s.t. } 0 \leq \phi_{n} \leq 2 \pi, \quad \forall n,
\end{gathered}
$$

where

$$
\boldsymbol{S}(\boldsymbol{\phi})=\sum_{k=1}^{K} \xi_{k} \tilde{\boldsymbol{H}}_{k} \tilde{\boldsymbol{\theta}}^{\mathrm{H}} \tilde{\boldsymbol{H}}_{k}^{\mathrm{H}}=\tilde{\boldsymbol{H}}\left(\left(\tilde{\boldsymbol{\theta}} \tilde{\boldsymbol{\theta}}^{\mathrm{H}}\right) \otimes \boldsymbol{\Xi}\right) \tilde{\mathbf{H}}^{\mathrm{H}},
$$

with $\xi_{k}=P_{k} / \sigma^{2}, \boldsymbol{\Xi}=\operatorname{diag}\left(\left[\xi_{1}, \xi_{2}, \cdots, \xi_{K}\right]^{\top}\right), \tilde{\mathbf{H}}=$ $\left[\tilde{\boldsymbol{H}}_{1}, \tilde{\boldsymbol{H}}_{2}, \cdots, \tilde{\boldsymbol{H}}_{K}\right] \in \mathbb{C}^{M \times K(N+1)}$, and $\otimes$ for the Kronecker product.

It should be noted that problem (7) with the unit modulus reflection is equivalent to a subproblem of the IRS-aided MIMO capacity problem, which was solved by sequentially optimizing an IRS element at a time while fixing the other elements in [16]. Unfortunately, the approach in [16] not only provides a suboptimal solution, but also leads to a computational delay due to sequential optimization. In the following, we will provide alternative methods providing either a better performance for the unit modulus reflection or a faster computation for the generalized reflection.

\section{IRS REFLECTION OPTIMIZATION}

\section{A. Determinant Maximization for Unit Modulus Reflection}

We first solve problem (7) for the unit modulus reflection through an extended SDR in a form of determinant maximization to obtain an upper bound on the optimal sum rate and a solution providing a performance close to the optimal one.

For this purpose, we define $\tilde{\boldsymbol{X}}=\tilde{\boldsymbol{\theta}} \tilde{\boldsymbol{\theta}}^{\mathrm{H}} \in \mathbb{S}_{+}^{N+1}$ and $\tilde{\mathcal{R}}(\tilde{\boldsymbol{X}})=\log _{2} \operatorname{det}\left(\boldsymbol{I}_{M}+\tilde{\mathbf{H}}(\tilde{\boldsymbol{X}} \otimes \boldsymbol{\Xi}) \tilde{\mathbf{H}}^{\mathrm{H}}\right)$ to transform (7) into

$$
\begin{aligned}
\max _{\tilde{\boldsymbol{X}} \in \mathbb{S}_{+}^{N+1}} & \tilde{\mathcal{R}}(\tilde{\boldsymbol{X}}) \\
\text { s.t. } & \tilde{X}_{n, n}=1, n=1,2, \cdots, N+1, \\
& \operatorname{rank}(\tilde{\boldsymbol{X}})=1
\end{aligned}
$$


where $\tilde{X}_{n, l}$ denotes the $(n, l)$ th entry of $\tilde{\boldsymbol{X}}$. If the rank-one constraint is ignored, problem (9) can be transformed into a convex optimization problem as

$$
\begin{aligned}
& \max _{\tilde{\boldsymbol{X}} \in \mathbb{S}_{+}^{N+1}, \boldsymbol{Y} \in \mathbb{S}_{+}^{M}} \log _{2} \operatorname{det} \boldsymbol{Y} \\
& \text { s.t. } \boldsymbol{I}_{M}+\tilde{\mathbf{H}}(\tilde{\boldsymbol{X}} \otimes \boldsymbol{\Xi}) \tilde{\mathbf{H}}^{\mathrm{H}} \succeq \boldsymbol{Y}, \\
& \tilde{X}_{n, n}=1, n=1,2, \cdots, N+1,
\end{aligned}
$$

which is known as the max-det problem defined in [21] since (10b) is a linear matrix inequality of semidefinite matrix $\tilde{\boldsymbol{X}}$. Thus, (10) can be solved with an existing convex optimization solver such as CVX and MOSEK to obtain the optimal solution $\tilde{\boldsymbol{X}}_{\diamond}$ and its corresponding optimal value $\tilde{\mathcal{R}}_{\diamond}$.

The optimal value $\tilde{\mathcal{R}}_{\diamond}$ obtained with relaxed constraints can serve as an upper bound on the optimal sum rate of problem (9). If $\tilde{\boldsymbol{X}}_{\diamond}$ is rank-one, the optimal solution $\boldsymbol{\theta}_{\diamond}$ is obtained from the unique eigenvector of $\tilde{\boldsymbol{X}}_{\diamond}$ with a nonzero eigenvalue. Otherwise, we obtain a feasible solution $\boldsymbol{\theta}_{\star}$ from $\tilde{\boldsymbol{X}}_{\diamond}$ through the Gaussian randomization procedure as in [10]. The randomization procedure generates $L$ randoms samples $\left\{\tilde{\boldsymbol{x}}_{l} \in \mathcal{C N}\left(0, \tilde{\boldsymbol{X}}_{\diamond}\right)\right\}_{l=1}^{L}$ to obtain the feasible solution candidates $\left\{\tilde{\boldsymbol{\theta}}_{l}\right\}_{l=1}^{L}$ by taking only the phases of the entries as $\theta_{l, n}=e^{j \angle \tilde{x}_{l, n}}$. We choose the best candidate as $\tilde{\boldsymbol{\theta}}_{\star}=$ $\max _{1 \leq l \leq L} \tilde{\mathcal{R}}\left(\tilde{\boldsymbol{\theta}}_{l} \tilde{\boldsymbol{\theta}}_{l}^{\mathrm{H}}\right)$ with which the IRS solution is obtained as $1 \leq l \leq L$

$\boldsymbol{\theta}_{\star}=\tilde{\theta}_{\star, N+1}^{*}\left[\tilde{\theta}_{\star, 1}, \tilde{\theta}_{\star, 2}, \cdots, \tilde{\theta}_{\star, N}\right]^{\top}$.

Remark 1: The max-det optimization shows a higher complexity than the SDR ranging from $\mathcal{O}\left(N^{3.5}\right)$ to $\mathcal{O}\left(N^{6.5}\right)$ [10], [14], which makes the computational time unacceptably high for a large $N$.

\section{B. L-BFGS Algorithm for Practical Reflection}

To tackle problem (7) for a large $N$ or for practical IRS reflection, we apply the L-BFGS algorithm [18], [19] developed for a large-scale problem with a complicated but a differentiable objective function. Instead of complex-valued variables $\boldsymbol{\theta}$, we adopt real-valued phases $\phi$ for optimization variables since constraints (7b) can be removed due the periodicity of the objective function. The L-BFGS algorithm starts with an initial estimate $\hat{\phi}_{0}$ of the optimal solution and proceeds to refine that estimate with a sequence of better estimates $\hat{\phi}_{t}, t=$ $1,2, \cdots$. The search direction for updating the estimates is identified with the gradient $\nabla \mathcal{R}(\phi)=\left[\frac{\partial \mathcal{R}}{\partial \phi_{1}}, \frac{\partial \mathcal{R}}{\partial \phi_{2}}, \cdots, \frac{\partial \mathcal{R}}{\partial \phi_{N}}\right]^{\top}$ of the objective function, which will be derived explicitly in the following.

Let us rewrite the objective function as $\mathcal{R}(\phi)=$ $\frac{1}{\ln 2} \ln \operatorname{det} \boldsymbol{Y}(\phi)$ with $\boldsymbol{Y}(\phi)=\boldsymbol{I}_{M}+\boldsymbol{S}(\phi)$. We then have

$$
\frac{\partial \mathcal{R}}{\partial \phi_{n}}=\frac{1}{\ln 2} \operatorname{tr}\left(\boldsymbol{Y}^{-1} \frac{\partial \boldsymbol{Y}}{\phi_{n}}\right)=\frac{1}{\ln 2} \operatorname{tr}\left(\boldsymbol{Y}^{-1} \frac{\partial \boldsymbol{S}}{\partial \phi_{n}}\right)
$$

from the differentiation of a determinant given by

$$
\frac{\partial \operatorname{det}(\boldsymbol{A})}{\partial t}=\operatorname{det}(\boldsymbol{A}) \operatorname{tr}\left(\boldsymbol{A}^{-1} \frac{\partial \boldsymbol{A}}{\partial t}\right) .
$$

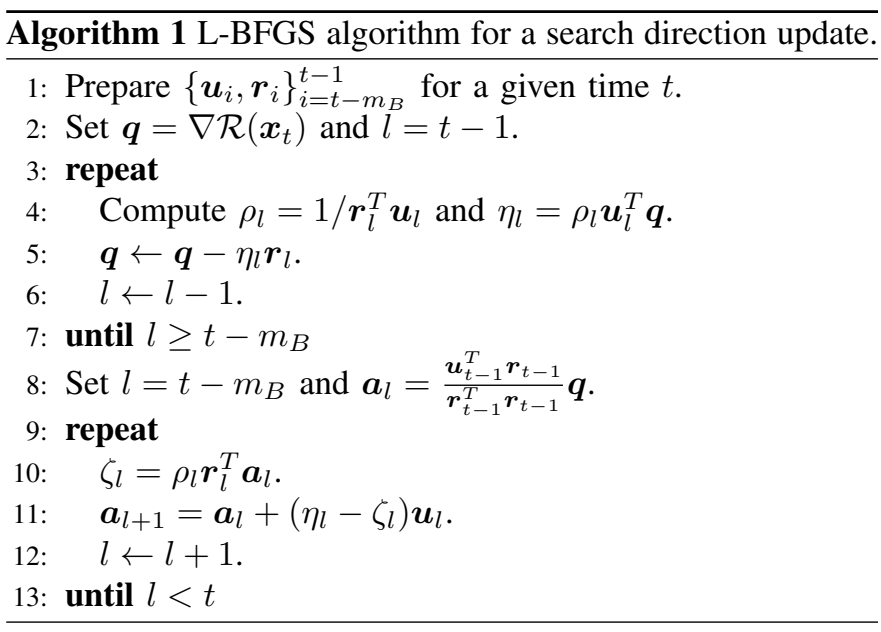

For explicit expression on (11), we rewrite $\boldsymbol{S}(\phi)$ in (8) as

$$
\begin{aligned}
\boldsymbol{S}(\boldsymbol{\phi}) & =\sum_{n=1}^{N} \sum_{\nu=1}^{N} \theta_{n} \theta_{\nu}^{*} \sum_{k=1}^{K} \xi_{k} \boldsymbol{h}_{k, n} \boldsymbol{h}_{k, \nu}^{\mathrm{H}}+\sum_{k=1}^{K} \xi_{k} \boldsymbol{v}_{k} \boldsymbol{v}_{k}^{\mathrm{H}} \\
& =\sum_{n=1}^{N}\left\{\theta_{n} \sum_{k=1}^{K} \xi_{k} \boldsymbol{h}_{k, n} \boldsymbol{v}_{k}^{\mathrm{H}}+\theta_{n}^{*} \sum_{k=1}^{K} \xi_{k} \boldsymbol{v}_{k} \boldsymbol{h}_{k, n}^{\mathrm{H}}\right\}
\end{aligned}
$$

where $\boldsymbol{h}_{k, n}$ being the $n$th column of $M \times N$ matrix $\boldsymbol{H}_{k}$. We then express (13) in matrix forms as

$$
\boldsymbol{S}(\phi)=(\boldsymbol{\Theta \mathcal { H }}+\mathcal{V}) \boldsymbol{\Xi}(\boldsymbol{\Theta} \mathcal{H}+\mathcal{V})^{\mathrm{H}},
$$

where $\boldsymbol{\Theta}=\boldsymbol{I}_{M} \otimes \boldsymbol{\theta}^{\top}, \mathcal{V}=\left[\boldsymbol{v}_{1}, \boldsymbol{v}_{2}, \cdots, \boldsymbol{v}_{K}\right]$, and

$\mathcal{H}=\left[\begin{array}{cccc}\boldsymbol{h}_{1,1} & \boldsymbol{h}_{2,1} & \cdots & \boldsymbol{h}_{K, 1} \\ \boldsymbol{h}_{1,2} & \boldsymbol{h}_{2,2} & \cdots & \boldsymbol{h}_{K, 2} \\ \vdots & \vdots & \ddots & \vdots \\ \boldsymbol{h}_{1, N} & \boldsymbol{h}_{2, N} & \cdots & \boldsymbol{h}_{K, N}\end{array}\right]=\left[\begin{array}{c}\mathcal{H}_{1} \\ \mathcal{H}_{2} \\ \vdots \\ \mathcal{H}_{N}\end{array}\right] \in \mathbb{C}^{M N \times K}$.

We than have as

$\frac{\partial \boldsymbol{S}}{\partial \phi_{n}}=\theta_{n}^{\prime} \mathcal{H}_{n} \boldsymbol{\Xi}(\boldsymbol{\Theta \mathcal { H }}+\mathcal{V})^{\mathrm{H}}+\left(\theta_{n}^{*}\right)^{\prime}(\boldsymbol{\Theta} \mathcal{H}+\mathcal{V}) \boldsymbol{\Xi} \mathcal{H}_{n}^{\mathrm{H}}$,

where $\theta_{n}^{\prime}=\frac{d \theta_{n}}{d \phi_{n}}$ with $\left(\theta_{n}^{*}\right)^{\prime}=\left(\theta_{n}^{\prime}\right)^{*}$. For practical IRS reflection (2), we have

$$
\theta_{n}^{\prime}=\left\{\beta_{n}^{\prime}\left(\phi_{n}\right)+j \beta_{n}\left(\phi_{n}\right)\right\} e^{j \phi_{n}},
$$

where

$$
\beta_{n}^{\prime}\left(\phi_{n}\right)=\frac{\alpha\left(1-\beta_{\min }\right)}{2}\left(\frac{\sin \left(\phi_{n}-\phi_{0}\right)+1}{2}\right)^{\alpha-1} \cos \left(\phi_{n}-\phi_{0}\right) .
$$

For the unit modulus reflection with $\alpha=0$ and $\beta_{\min }=0$, (17) becomes $\theta_{n}^{\prime}\left(\phi_{n}\right)=j e^{j \phi_{n}}$. With (16) in the form of $\frac{\partial \boldsymbol{S}}{\partial \phi_{n}}=$ $\boldsymbol{B}+\boldsymbol{B}^{H}$, we can compute (11) as

$$
\frac{\partial \mathcal{R}}{\partial \phi_{n}}=\frac{2}{\ln 2} \Re\left\{\theta_{n}^{\prime} \operatorname{tr}\left(\boldsymbol{Y}^{-1} \mathcal{H}_{n} \boldsymbol{\Xi}(\boldsymbol{\Theta \mathcal { H }}+\mathcal{V})^{\mathrm{H}}\right\},\right.
$$

where $\Re\{\cdot\}$ represents the real part.

The L-BFGS algorithm with $m_{B}$ memories [18], [19] stores the sequences $\left\{\boldsymbol{u}_{i}, \boldsymbol{r}_{i}\right\}_{i=t-m_{B}}^{t-1}$ for a given time $t$, where $\boldsymbol{u}_{i}=$ $\phi_{i+1}-\phi_{i}$ is the variable variation and $\boldsymbol{r}_{i}=\nabla \mathcal{R}\left(\phi_{i+1}\right)-$ $\nabla \mathcal{R}\left(\phi_{i}\right)$ is the gradient variation. At a given time $t$, the LBFGS finds the ascent direction $\boldsymbol{a}_{t}$ as in Algorithm 1. 


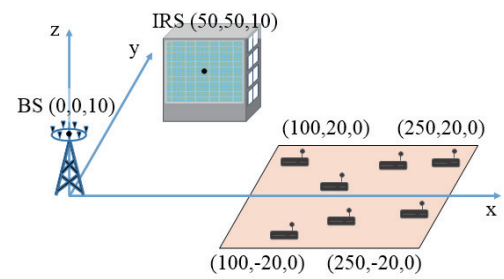

Fig. 2. Simulation setup for the uplink IRS-aided NOMA.

Remark 2: The computational complexity of the L-BFGS algorithm is given by $\mathcal{O}\left(\left(M^{3}+M^{2} N K+m_{B} N\right) I_{\text {prop }}\right)$ with $I_{\text {prop }}$ iterations up to a convergence since the algorithm computes $3 M^{3}+M^{2} N K$ complex multiplications for each gradient and $4 m_{B} N+N$ multiplications for the update in the search direction. If the sequential phase optimization in [16] is applied by assuming the unit modulus reflection, its computational complexity is given by $\mathcal{O}\left(\left(M^{3}+M K\right) N I_{\text {conv }}\right)$ with $I_{\text {conv }}$ iterations of updating $N$ elements requiring a matrix inversion and an eigenvalue decomposition per element.

\section{Simulation Results}

We evaluate the performance of the uplink IRS-aided NOMA when the maximum transmit power and noise power are set to $P_{k}^{\max }=23 \mathrm{dBm}$ for $k \in \mathbb{K}$ and $\sigma^{2}=-100$ $\mathrm{dBm}$, respectively. We set $L=50$ for Gaussian randomization, $m_{B}=10$ for the L-BFGS algorithm, and $10^{-5}$ for the error tolerance of all algorithms. We choose $\alpha=1.6, \beta_{\min }=0.2$, and $\phi_{0}=0.43 \pi$ for practical IRS reflection as in [17]. The simulation setup is illustrated in Fig. 2, where the $(x, y, z)$ coordinates are given in meter. The BS and IRS are located at a fixed point at $(0,0,10)$ and $(50,50,10)$, respectively, whilst the devices are uniformly distributed in the shaded rectangular region.

The channels are modelled as

$$
\begin{aligned}
& \boldsymbol{G}=\sqrt{\frac{\kappa_{\mathrm{R}, \mathrm{B}} \omega_{\mathrm{R}, \mathrm{B}}}{\kappa_{\mathrm{R}, \mathrm{B}}+1}} \boldsymbol{G}_{\mathrm{LoS}}+\sqrt{\frac{\omega_{\mathrm{R}, \mathrm{B}}}{\kappa_{\mathrm{R}, \mathrm{B}}+1}} \boldsymbol{G}_{\mathrm{NLoS}}, \\
& \boldsymbol{f}_{k}=\sqrt{\frac{\kappa_{k, \mathrm{R}} \omega_{k, \mathrm{R}}}{\kappa_{k, \mathrm{R}+1}}} \boldsymbol{f}_{\mathrm{LoS}, k}+\sqrt{\frac{\omega_{k, \mathrm{R}}}{\kappa_{k, \mathrm{R}+1}}} \boldsymbol{f}_{\mathrm{NLoS}, k}, k \in \mathbb{K}, \\
& \boldsymbol{v}_{k}=\sqrt{\frac{\kappa_{k, \mathrm{~B}} \omega_{k, \mathrm{~B}}}{\kappa_{k, \mathrm{~B}+1}}} \boldsymbol{v}_{\mathrm{LoS}, k}+\sqrt{\frac{\omega_{k, \mathrm{~B}}}{\kappa_{k, \mathrm{~B}}+1}} \boldsymbol{v}_{\mathrm{NLoS}, k}, k \in \mathbb{K},
\end{aligned}
$$

where the subscripts LoS and NLoS represent the line-ofsight (LoS) and non-LoS (NLoS) components, respectively, and $\omega_{x, y}$ and $\kappa_{x, y}$ denote the path loss and Rician factor between nodes $x$ and $y$ for $x, y \in \mathbb{K} \cup\{\mathrm{R}, \mathrm{B}\}$; R denotes the IRS and B denotes the BS. The path loss is given by $\omega_{x, y}=$ $10^{-3} d_{x, y}^{-\nu_{x, y}}$ at distance $d_{x, y}$ with path loss exponent $\nu_{x, y}$. We set $\nu_{\mathrm{R}, \mathrm{B}}=2.2, \nu_{\mathrm{R}, k}=2.8, \nu_{k, \mathrm{~B}}=4, \kappa_{\mathrm{R}, \mathrm{B}}=2=\kappa_{\mathrm{R}, k}=2$, and $\kappa_{\mathrm{B}, k}=0$ for $k \in \mathbb{K}$. Assuming a uniform linear array for the BS antennas and an $N_{v} \times N_{h}$ uniform planar array for the IRS, the LoS components are given by [16]

$$
\begin{aligned}
& \boldsymbol{G}_{\mathrm{LoS}}=\boldsymbol{a}_{\mathrm{B}}\left(\varphi_{\mathrm{R}, \mathrm{B}}^{A}\right) \boldsymbol{a}_{\mathrm{R}}^{\mathrm{H}}\left(\varphi_{\mathrm{R}, \mathrm{B}}^{D}, \vartheta_{\mathrm{R}, \mathrm{B}}^{D}\right), \\
& \boldsymbol{f}_{\mathrm{LoS}, k}=\boldsymbol{a}_{\mathrm{R}}\left(\varphi_{k, \mathrm{R}}^{A}, \vartheta_{k, \mathrm{R}}^{A}\right), \quad k \in \mathbb{K}
\end{aligned}
$$

where $\boldsymbol{a}_{\mathrm{B}}(\varphi)=\left[1, e^{j \pi \sin (\varphi)}, \cdots, e^{j \pi(M-1) \sin (\varphi)}\right]^{T}$ is the array response at the BS with the half-wavelength antenna spac-

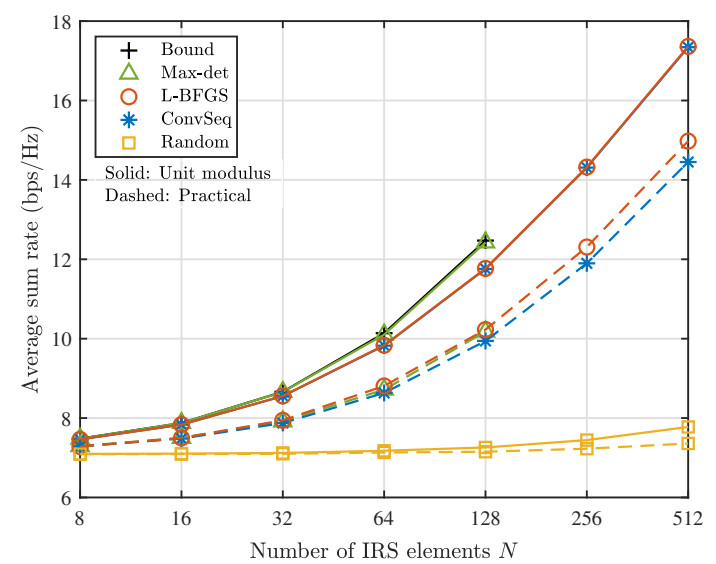

Fig. 3. Average sum rate as the number $N$ of IRS elements increases when $M=2$ and $K=4$.

ing, $\boldsymbol{a}_{\mathrm{R}}(\varphi, \vartheta) \in \mathbb{C}^{N}$ is the array response at the IRS as specified in [16], $\varphi_{x, y}^{A}\left(\vartheta_{x, y}^{A}\right)$ and $\varphi_{x, y}^{D}\left(\vartheta_{x, y}^{D}\right)$ are the azimuth (elevation) angle-of-arrival (AoA) and angle-of-departure (AoD) from $x$ to $y$, respectively. The LoS components are determined by the location of nodes in the network with $N_{v}=8$ and $N_{h}=N / N_{v}$ elements in the vertical and horizontal axes of the IRS, respectively. The NLoS components are modelled by uncorrelated Gaussian random variables.

The average sum rate of the network is shown as the number $N$ of IRS elements increases in Fig. 3 when $M=2$ and $K=4$ for both unit modulus and practical IRS reflection cases indicated by the solid and dashed lines, respectively. The performance of L-BFGS is evaluated by applying the L-BFGS algorithm with the gradient derived for each of the unit modulus and practical reflection cases. Max-det and ConvSeq obtain the solutions for the unit modulus reflection as in Subsection III-A and as in [16], respectively, which are also applied for the practical reflection to obtain the sum rate. The optimal value of the max-det problem (10) is denoted by Bound to serve as an upper bound on the optimal performance with the unit modulus reflection. The performance with random IRS phases is also shown to serve as a lower bound.

Clearly, the average sum rate increases steeply with $N$ by applying an optimization method. With the unit modulus reflection, Max-det provides the best performance close to the optimal one estimated by Bound while L-BFGS and ConvSeq performing almost identically exhibits a loss over Max-det. However, the performance of Max-det is shown up to $N=128$ due to its computational overhead increasing with $N$. The ratio of the computational time of L-BFGS over that of Maxdet is given by $5.5 \%, 0.86 \%$, and $0.10 \%$ for $N=32,64$, and 128, respectively. For the practical reflection, L-BFGS provides the best performance by considering the effect of phase-dependent amplitudes in finding the next search point. In addition, the ratio of the computational time of L-BFGS over that of ConvSeq is given by $18 \%, 9.1 \%$, and $5.3 \%$ for $N=128,256$, and 512 , respectively.

Fig. 4 compares the average sum rate as the number $K$ of devices increases when $N=64$ with $M=2$ in Fig. 4(a) and with $M=4$ in Fig. 4(b). It is obvious that the average sum rate 

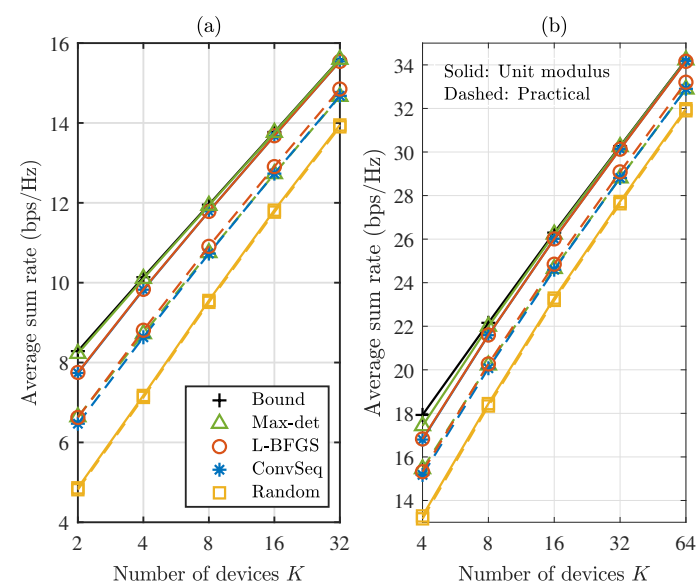

Fig. 4. Average sum rate as the number $K$ of devices increases when $N=64$ : (a) $M=2$ (b) $M=4$.
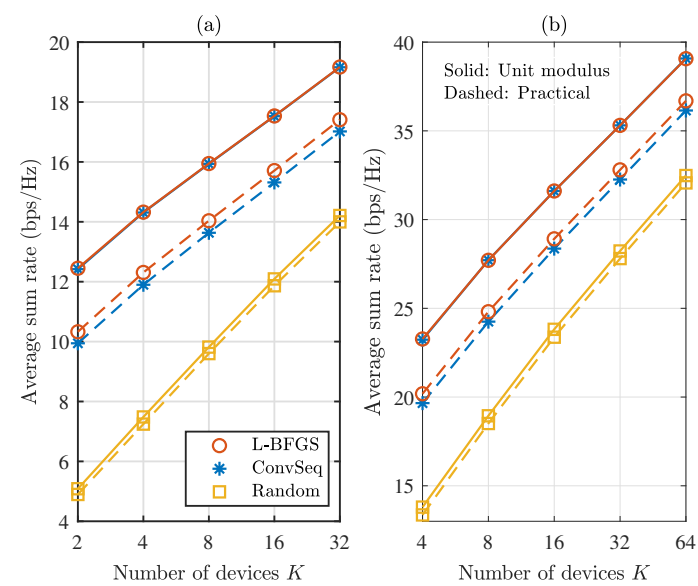

Fig. 5. Average sum rate as the number $K$ of devices increases when $N=$ 256: (a) $M=2$ (b) $M=4$.

increases with the number $K$ of devices. The results with unit modulus reflection show that Max-det outperforms L-BFGS and ConvSeq for a relatively small $K$ but three algorithms perform similarly achieving the optimal performance as $K$ increases. The average sum rate is almost doubled due to multiplexing gain when the number of antennas is increased from $M=2$ in Fig. 4(a) to $M=4$ in Fig. 4(b). Practical IRS reflection incurs a performance loss which tends to be smaller as the number of devices or the number of antennas increases. An infinitesimal gain of L-BFGS over the other methods is observed for the practical reflection with $N=64$.

The performance obtained with $N=256$ is shown in Fig. 5 when the other condition remain unchanged from Fig. 4. The results of Max-det and Bound are not provided due to their complexity. As $N$ increases from 64 in Fig. 4 to 256 in Fig. 5, the gain of an IRS optimization over Random increases significantly. L-BFGS provides a similar or slightly improved performance compared with ConvSeq, which is obtained at a computational time about $11 \%$ and $18 \%$ of ConvSeq with $M=2$ and 4, respectively, for most of $K$ values in the figure. The results confirm the advantages of L-BFGS over ConvSeq for a larger $N$ and also with practical reflection.

\section{CONCLUding Remarks}

We have considered a sum rate maximization problem for the IRS-aided uplink multiantenna NOMA with the generalized reflection from the unit modulus to phase-dependent amplitudes. We have solved the problem under the unit modulus reflection through extended SDR of max-det problem to obtain an upper bound on the sum rate and a near-optimal solution for a small $N$ of IRS elements. We have applied the L-BFGS algorithm for the generalized reflection by providing the gradient in an explicit form. The results showed that LBFGS provides a competitive solution for a large $N$ in both computational time and performance.

\section{REFERENCES}

[1] C. Huang et al., "Holographic MIMO surfaces for 6G wireless networks: Opportunities, challenges, and trends," IEEE Wirel. Commun., vol. 27, no. 5, pp. 118-125, Oct. 2020.

[2] Q. Wu, S. Zhang, B. Zheng, C. You, and R. Zhang, "Intelligent reflecting surface aided wireless communication: A tutorial," IEEE Trans. Commun., vol. 69, no. 5, pp. 3313-3351, May 2021.

[3] J. Choi, "NOMA-Based random access With multichannel ALOHA," IEEE J. Select. Areas Commun., vol. 35, pp. 2736-2743, Dec. 2017.

[4] Z. Ding and H. V. Poor, "A simple design of IRS-NOMA transmission," IEEE Commun. Lett., vol. 24, no. 5, pp. 1119-1123, May 2020.

[5] B. Zheng, Q. Wu, and R. Zhang, "Intelligent reflecting surface-assisted multiple access with user pairing: NOMA or OMA?" IEEE Commun. Lett., vol. 24, no. 4, pp. 753-757, Apr. 2020.

[6] X. Mu, Y. Liu, L. Guo, J. Lin, and N. Al-Dhahir, "Capacity and optimal resource allocation for IRS-assisted multi-user communication systems," IEEE Trans. Commun., vol. 69, no. 6, pp. 3771-3786, June 2021.

[7] X. Mu, Y. Liu, L. Guo, J. Lin, and N. Al-Dhahir, "Exploiting intelligent reflecting surfaces in NOMA networks: Joint beamforming optimization," IEEE Trans. Wirel. Commun., vol. 19, no. 10, pp. 6884-6898, Oct. 2020.

[8] F. Fang, Y. Xu, Q. V. Pham, and Z. Ding, "Energy-efficient design of IRS-NOMA networks," IEEE Trans. Veh. Techn., vol. 69, no. 11, pp. 14088-14092, Nov. 2020.

[9] J. Zhu, Y. Huang, J. Wang, K. Navaie, and Z. Ding, "Power efficient IRS-assisted NOMA," IEEE Trans. Commun., vol. 69, no. 2, pp. 900913, Feb. 2021.

[10] G. Yang, X. Xu, Y. Liang, and M. Renzo, "Reconfigurable intelligent surface-assisted non-orthogonal multiple access," IEEE Trans. Wirel. Commun., vol. 20, no. 5, pp. 3137-3151, May 2021.

[11] X. Xie, F. Fang, and Z. Ding, "Joint optimization of beamforming, phase-shifting and power allocation in a multi-cluster IRS-NOMA network," IEEE Trans. Veh. Techn., vol. 70, pp. 7705-7717, Aug. 2021.

[12] M. Zeng, X. Li, G. Li, W. Hao, and O. A. Dobre, "Sum rate maximization for IRS-assisted uplink NOMA," IEEE Commun. Lett., vol. 25, no. 1, pp. 234-238, Jan. 2021.

[13] D. Song, W. Shin, and J. Lee, "A maximum throughput design for wireless powered communication networks with IRS-NOMA," IEEE Wirel. Commun. Lett., vol. 10, no. 4, pp. 849-853, Apr. 2021.

[14] Q. Wu, X. Zhou, and R. Schober, "IRS-assisted wireless powered NOMA: Do we really need different phase shifts in DL and UL?," IEEE Wirel. Commun. Lett., vol. 10, no. 7, pp. 1493-1497, July 2021.

[15] B. Lyu et al., "IRS-Assisted downlink and uplink NOMA in wireless powered communication networks," IEEE Trans. Veh. Techn., vol. 71, no. 1, pp. 1083-1088, Jan. 2022.

[16] S. Zhang and R. Zhang, "Capacity characterization for intelligent reflecting surface aided MIMO communication," IEEE J. Selct. Areas Commun., vol. 38, no. 8, pp. 1823-1838, Aug. 2020.

[17] S. Abeywickrama, R. Zhang, Q. Wu, and C. Yuen, "Intelligent reflecting surface: practical phase shift model and beamforming optimization," IEEE Trans. Commun., vol. 68, no. 9, pp. 5849-5863, Sept. 2020.

[18] D. C. Liu and J. Nocedal, "On the limited memory BFGS method for large scale optimization," Mathematical Programming, vol. 45, no. 1-3, pp. $503-528,1989$.

[19] W. Chen, Z. Wang, and J. Zhou, "Large-scale L-BFGS using MapReduce," Proc. NIPS, vol. 1, pp. 1332-1340, Dec. 2014.

[20] D. Tse and P. Viswanath, Fundamentals of Wireless Communication, Cambridge University Press, 2005.

[21] S. Boyd and L. Vandenberghe, Convex Optimization, Cambridge Univ. Press, Cambridge, UK, 2004. 